Voyage au pays des Lau (îles Salomon, début du XXI siècle). Le déclin d'une gynécocratie, de Pierre MARANDA

\title{
Raymond MAYER
}

\section{OpenEdition}

\section{Journals}

Édition électronique

URL : http://journals.openedition.org/jso/5839

DOI : $10.4000 /$ jso.5839

ISSN : 1760-7256

Éditeur

Société des océanistes

\section{Édition imprimée}

Date de publication : 30 juin 2009

Pagination : 173-174

ISBN : 978-2-85430-024-6

ISSN : 0300-953x

\section{Référence électronique}

Raymond MAYER, «Voyage au pays des Lau (îles Salomon, début du xxi siècle). Le déclin d'une

gynécocratie, de Pierre mARANDA », Journal de la Société des Océanistes [En ligne], 128 | janvier-juin 2009, mis en ligne le 05 mai 2009, consulté le 21 septembre 2020. URL : http://journals.openedition.org/jso/ 5839 ; DOI : https://doi.org/10.4000/jso.5839 
Guinée et à la Mélanésie insulaire, avec une qualité d'information et une richesse de vues devant intéresser même les connaisseurs. Les notices des pièces exposées, rédigées par Frank Herreman en personne, méritent elles aussi d'être lues de près, pour les renseignements qu'elles reproduisent, généralement avec prudence, ou les observations qu'elles formulent, parfois de nature à rafraîchir le regard porté sur les objets.

À propos d'un superbe « idiophone à friction » de Nouvelle-Irlande ( $\mathrm{n}^{\circ} 83$, p. 96), objet en mains privées et probablement inédit, sauf à le rechercher dans la (très recommandable et utile) base de données de photographies d'étude du Metropolitan Museum de New York ou les images prises chez Conru Primitive, le marchand qui le proposait à l'International Tribal Antiques Show de New York en mai 2000, Frank Herreman fait ainsi observer que si la tradition et les spécialistes reconnaissent dans cet instrument (nommé livika dans l'aire linguistique mandak) « la forme d'un oiseau, on y distingue pourtant des éléments empruntés à d'autres animaux. Le tambour est par exemple doté de dents, ce que les oiseaux n'ont évidemment pas "). Michael Gunn avait déjà formulé cette remarque élémentaire (1997 : 92), mais sans la reprendre dans le texte qu'il a récemment consacré à ce type d'objet (2007 : 192). Il est heureux que le présent catalogue, dans cette courte notice comme dans d'autres, rappelle les incertitudes entourant ces objets. Mais, pour les connaisseurs et les spécialistes, son intérêt principal réside évidemment dans le nombre important (136 sur 198) de pièces provenant de collections privées et pour beaucoup inédites. Pour s'en tenir ici à l'exemple précédemment évoqué, peu de musées conservent un livika d'une ornementation aussi riche et intéressante, et sa publication officielle ne peut qu'encourager de nouvelles études sur ce « violon des morts » de Nouvelle-Irlande.

\section{RÉFÉRENCES CITÉES}

GunN Michael, 1997. Arts rituels d'Océanie. NouvelleIrlande dans les collections du musée BarbierMueller, Milan, Skira.

GunN Michael et Philippe Peltier (éds), 2007. Nouvelle-Irlande, Arts du Pacifique Sud, Paris, MQB-5 continents.

Metropolitan Museum, base de données, http:// www.metmuseum.org/works_of_art/collection_ database/all/friction_drum_livikia_kevin_conru/.

\section{Gilles BounOuRE}

Maranda Pierre, 2008. Voyage au pays des Lau (îles Salomon, début du XXI siècle). Le déclin d'une gynécocratie, Paris, éditions Cartouche, 189 p., bibliogr., 1 carte, dessins noir et blanc.

Il est probablement tabou de référer trop ouvertement un ouvrage à un autre, mais il est évident que Tristes tropiques a fait des émules et qu'un long com- pagnonnage intellectuel avec Lévi-Strauss a fait forcément école, même si c'est partiellement sur des conclusions opposées. Le récit de Pierre Maranda ne commence pas par la formule « je hais les voyages », mais au contraire invite explicitement au voyage, en fournissant notamment, sous la forme de «conseils pratiques » (pp. 173-175), un séjour quasi clés en mains à chaque lecteur qui voudrait tenter l'aventure ! On a envie de dire que le véritable titre de ce livre grand public pourrait être «Joyeux tropiques» plutôt que "déclin d'une gynécocratie», tant la tonalité de l'ouvrage restitue une joie de vivre autant qu'une vision du monde et tant la partie théorique se fond dans la narration du premier séjour en famille de l'auteur dans la lagune des Lau en 1966. L'invitation au voyage est d'ailleurs soulignée par le parti pris de l'éditeur d'agrémenter l'ouvrage par des illustrations de style BD plutôt que par des photographies. Ainsi s'écrirait la légende des « anthropologies premières $»$ !

Celle-ci rappelle, sur un mode narratif que seul autorise l'âge de la sagesse, les préjugés communs qui pouvaient noyer, autant chez les administrateurs coloniaux que chez les anthropologues eux-mêmes, la volonté de s'établir durablement au sein d'une population dont on ne parlait pas la langue et dont on ne partageait pas la culture. On fait donc le tour d'un îlot (pp. 36-46) avec l'auteur, sa femme et leur fils de deux ans, pour découvrir les us et coutumes d'une population dont on apprend progressivement à connaître les rudiments de leurs croyances religieuses (pp. 46-49), de leur histoire (pp. 55-58), en particulier de leur célèbre établissement en îlots artificiels (pp. 58-69), de l'arrivée du christianisme (pp. 79-85), de l'économie de leurs marchés locaux (pp. 87-99), accessoirement de leurs pratiques sexuelles (pp. 125-142), et enfin du passage à l'indépendance (pp. 151-161). Les intitulés des chapitres ou leurs intertitres sont parfois racoleurs - «Vivre parmi des cannibales ?», « Travaux pratiques en érotisme à l'usage des hommes »-, parfois tendrement familiers - « Sages, les Lau ne pressent pas le citron quand il s'agit de la synergie mer-terre », « Les travaux et les jours - sans s'en faire », " Pêcher sans se fatiguer»-, parfois plus ironiques - «Les chrétiens en quarantaine », "Une indépendance de patate douce ». Le procédé est quelque peu rustique, mais efficace. Le récit se lit d'une traite.

Reste la question préjudicielle qui encadre le récit, qui en fait pratiquement son inclusio, et qui est manifestement mise en exergue à la fois par l'auteur et par l'éditeur (pp. 11-18) : celle de la mise en accusation publique de l'anthropologue au terme de ses entreprises. Le problème est résumé en deux phrases reproduites en couverture :

« Les Lau attendaient depuis près d'un demi-siècle l'arrivée de "gens du bout de la mer" quand Pierre Maranda débarqua un jour de 1966. [... Quarante ans plus tard] on l'accusa d'avoir dérobé l'Esprit Pieuvre tutélaire du clan, de le séquestrer dans sa piscine à Québec et d'être devenu grâce à lui millionnaire!»

À travers une sorte de mémoire en défense de son expérience particulière, qui peut paraître à certains 
égards bien cruelle, le grand anthropologue qu'est Pierre Maranda annonce probablement plus que son livre n'en écrit. Ce livre est en effet plus qu'un aveu à la Costa-Gavras ou une confession à la Rousseau. Son récit et son annexe sur les conditions contemporaines d'accès aux lieux et aux personnes des Lau ne font que renforcer la douloureuse impression que l'on retire sur les singulières circonstances de l'ostracisme qu'il est amené à vivre, quarante ans après ses magnifiques « premiers terrains ». Voici un anthropologue qui aura vécu à la fois l'endroit et l'envers d'un même terrain, étant passé d'un accueil extrêmement favorable à une réprobation radicale valant interdiction de séjour.

On doit lui reconnaître une honnêteté respectable à confesser le sort (avec la polysémie très anthropologique qui sied à ce terme) qu'il subit. Je tiens pourtant ce livre pour l'enterrement à la fois symbolique et réel des pratiques anthropologiques des $\mathrm{XIX}^{\mathrm{e}}$ et $\mathrm{XX}^{\mathrm{e}}$ siècles. Il inaugure peut-être même une révolution dans la discipline, qui n'est sans doute pas encore bien perçue par les professionnels, mais dont les signes montent progressivement en puissance jusqu'à l'exacerbation manifeste. De quoi s'agit-il ? Des droits et des devoirs $\mathrm{du}$ « terrain », j'emploie le singulier à dessein.

Il paraît évident à tout anthropologue que le devoir absolu d'avoir à "faire du terrain » s'accompagne symétriquement d'un imprescriptible droit d'accès. Or rien n'est moins sûr, ou ne l'est plus du tout. Malgré toutes les dénégations théoriques de l'auto-proclamée « observation participante » (Godelier, JSO 2007-2), le terrain ethnographique peut apparaître (et transparaît ainsi dans maintes pratiques effectives) comme une forme d'agression. La violence exercée sur terrain adverse n'a d'équivalent que le retour du boomerang. Dans l'expérience de Pierre Maranda, la chance du premier terrain (un providentiel don du ciel et la faveur de mythes historiques que l'on croirait effectivement sortis d'un ouvrage de Marshall Sahlins) n'a d'égale que la malchance de l'après-terrain. La symétrie est parfaite.

Mais on peut aller plus loin que d'en référer à un hasard culturel. Une histoire structurelle est peut-être en train de s'écrire sur le dos de l'anthropologie, indépendamment des anthropologues qui en font les frais. Autrement dit, ce n'est pas tant le comportement personnel de l'anthropologue qui est à mettre en cause, que le principe de la pratique anthropologique ellemême. Il est évident que tous les congrès d'anthropologues qui se sont penchés, depuis les années 1980, sur les nouvelles conditions d'accès au terrain, conditions résultant en particulier des indépendances, sont dans l'obligation non seulement d'entériner les situations de blocage, mais de renouveler leur approche du problème.

La nouveauté est celle-ci : l'accès symétrique, par les populations rencontrées, aux informations scientifiques et la prise de conscience de l'inégalité des statuts entre le chercheur et le " cherché » sont quelques-unes des clés qui permettent de comprendre l'évolution des problèmes et de leurs solutions. La nouvelle formulation des problèmes apporte en même temps une nouvelle forme de solution. Le fait que chaque culture produise maintenant ses universitaires n'a pas encore retenu l'attention des praticiens actuels, et pourtant on doit s'attendre à ce que ses effets soient non seulement locaux, mais bien globaux.

Raymond MAYER,

Université Omar Bongo de Libreville et Université de Lyon 2

\section{McFadgen Bruce, 2007. Hostile Shores. Catastrophic Events in Prehistoric New Zealand and their Impact on Maori Coastal Communities, Auckland Uni- versity Press, Auckland. xiv-298 p., appendices, bibliogr., index, nombreuses ill. noir et blanc, cartes et figures dans le texte.}

Dû à un archéologue chevronné, auteur de plus de soixante-dix contributions scientifiques tirées de quatre décennies de travail de fouille et d'analyse des données de terrain, ce livre a deux ambitions explicites, exposées dans son sous-titre et rappelées p. $15:$ :en premier lieu décrire les diverses catastrophes naturelles » anciennes survenues en Nouvelle-Zélande « dans leur contexte stratigraphique pour corréler et dater ces événements préhistoriques » et « en second lieu explorer les effets de ces événements sur les communautés littorales préhistoriques ». Sur ces deux sujets formant aussi le plan du livre, il apporte à la fois des connaissances de première main et de remarquables exposés synthétisant avec grande clarté les progrès récents de l'archéologie néo-zélandaise. Mais la conclusion qu'il finit par en tirer semblera considérable à quiconque connaît un peu la civilisation et les arts maori, et le caractère encore énigmatique de leur évolution.

On l'oublie trop souvent, la Nouvelle-Zélande reste le théâtre de phénomènes volcaniques et sismiques intenses, du fait de sa situation à la jointure de deux plaques tectoniques, l'indo-australienne et la pacifique, le long d'une zone de subduction que prolongent notamment les fosses sous-marines de Puységur au sud et des Kermadec au nord. À côté de manifestations telluriques spectaculaires et quelquefois terrifiantes rapportées par les voyageurs ou les journalistes, rien n'est plus impressionnant que la carte dressée en 1952 par Harold Wellman (p. 21), montrant, d'après leurs correspondances géologiques, le glissement horizontal sur $480 \mathrm{~km}$ des parties est et ouest de l'île du Sud le long de la «faille alpine» qui la traverse entièrement. L'île du Nord comporte également diverses failles de même orientation, elles aussi à l'origine d'éruptions et de séismes, mais, selon l'auteur, les populations maori d'abord établies sur les côtes avaient moins à craindre de ces phénomènes que des tsunamis engendrés par l'activité tectonique sous-marine, et dont l'archéologie met en évidence les ravages répétés sur le littoral et les estuaires qui le ponctuent.

Voilà pourquoi, dans cette Nouvelle-Zélande qui peut être considérée globalement comme un «lieu précaire de résidence » (titre du premier chapitre), les Maori qui se fixèrent d'abord sur ses rivages empoissonnés, giboyeux et relativement fertiles, en vinrent à s'éloigner de ces côtes devenues « hostiles » au terme 